\title{
GIST Grade 2
}

National Cancer Institute

\section{Source}

National Cancer Institute. GIST Grade 2. NCI Thesaurus. Code C160733.

A grade that may refer to GISTs with malignant histologic characteristics. 